\title{
The immediate effects of local vibration on ankle plantar flexor muscle activation and peak torque in healthy adults
}

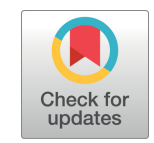

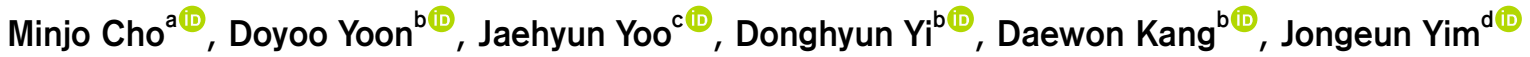 \\ ${ }^{a}$ Department of Physical Therapy, School of Health Science, Touro College, New York, USA \\ ${ }^{\mathrm{b}}$ Department of Physical Therapy, Graduate School of Sahmyook University, Seoul, Republic of Korea \\ 'Department of Health \& Human Performance, Sahmyook University, Seoul, Republic of Korea \\ ${ }^{\mathrm{d}}$ Department of Physical Therapy, College of Health Science and Social Welfare, Sahmyook University, Seoul, Republic of Korea
}

Objective: The vibration device is one of the most commonly used warm-up devices not only for healthy athletes but also for healthy individuals. Therefore, this study aimed to investigate the immediate effects of local vibration on ankle plantar flexor muscle activation and peak torque in healthy adults.

Design: One-group pretest-posttest design.

Methods: This was a single-group study comprising a total of 36 (16 males and 20 females) participants. The average age of the 36 participants was 22.3 years. All the participants' concentric and eccentric peak torques of the gastrocnemius lateralis muscle were measured using an isokinetic device. Simultaneously, the participants' muscle activity was measured by surface electromyography. After the pre-experimental data were collected, the participants comfortably sat on the prepared chair with their hips and knees flexed to $90^{\circ}$. While in sitting position, local vibration was applied for 10 minutes using a 1:1 ratio intermittent pulsing mode device based on a previous study. Then, the post-experimental data were collected immediately after the local vibration by performing a similar process performed during the pre-experimental data collection.

Results: The results showed a significant difference in muscle activity and eccentric peak torque $(p<0.05)$. On the contrary, concentric peak torque values showed an insignificant difference with pre- and post-value.

Conclusions: The results of this study demonstrated that local vibration can be possibly considered as one of the effective ways to increase ankle plantar flexor muscle activity and muscle performance, specifically the eccentric peak torque, in healthy adults.

Key Words: Electromyograpy, Gastrocnemius muscle, Muscle strength, Power, Vibration

\section{Introduction}

Since the inception of the new millennium the use and application of vibration therapy for medical, clinical, and rehabilitative purposes has been consistently developed [1]. A mechanical stimulus characterized by an oscillatory motion is referred to as vibration that physiologically stimulates the human body to induce changes and achieve treatment goals [2]. The intensity of vibration is determined by its own biomechanical variables' frequency and amplitude. In oscillatory motion, extension range determines the amplitude (peak to peak displacement, in $\mathrm{mm}$ ) of the vibration. The repetition rate of the cycles of oscillation determines the frequency of the vibration measured in hertz (Hz) [3]. Scientific objectives include the long-term and short-term effects of vibration therapy on gross motor function, strength, gait, and postural control, and the vibration therapy's effects on the bones and muscles [4]. Vibration therapy not only enhances muscle strength, power, joint position sense, blood flow under the skin [5], and act on human neuromuscular structure [6-8], but also effectively treats delayed onset muscle soreness [9]. Additionally, studies have shown that vibration therapy can

Received: 27 April, 2020 Revised: 2 June, 2020 Accepted: 3 June, 2020

Corresponding author: Jongeun Yim (ORCID https://orcid.org/0000-0001-7510-8233)

Department of Physical Therapy, College of Health Science and Social Welfare, Sahmyook University, 815 Hwarang-ro, Nowon-gu, Seoul 01795 , Republic of Korea Tel: 82-2-3399-1635 Fax: 82-2-3399-1639 E-mail: jeyim@syu.ac.kr

(c) This is an Open-Access article distributed under the terms of the Creative Commons Attribution Non-Commercial License (http://creativecommons.org/licenses/ by-nc/4.0) which permits unrestricted non-commercial use, distribution, and reproduction in any medium, provided the original work is properly cited.

Copyright @ 2020 Korean Academy of Physical Therapy Rehabilitation Science 
enhance motoneuron excitability.

Vibration therapy can be performed in two different ways. First, vibration therapy can be indirectly performed using vibrating platforms, dumbbells, and vibratory devices that are fixed to resistance training machines [1]. Second, vibration therapy can be directly performed in muscle bellies and/or tendons [10].

Regarding indirect vibration therapy, whole-body vibration (WBV) is the most commonly used type of vibration training [11]. Furthermore, in recent years, there has been an increased interest in the use of WBV because it not only increases athletic performance but also relates to untrained adults or recreational athletes $[12,13]$. However, WBV training has been widely used in sports medicine and rehabilitation [14]. However, WBV programs may not be suitable to everyone. Certainly, some participants are unable to bear weight because of the following reasons: (1) long-term immobilization (lower limb trauma), (2) immobilization by a cast, or (3) being in the early rehabilitation period after surgery (anterior cruciate ligament reconstruction). WBV participants will be stimulated three-dimensionally when standing or squatting on a vibration platform [15]. When vibration is activated, it begins to stimulate the muscle spindles and activates the alpha ( $\alpha$ )-motoneurons. While the exact underlying mechanisms of vibration remain unclear, reflex muscular contractions have been proposed [16]. A previous study has shown almost identical latency responses between the actual stretch reflexes and WBV-induced reflexes [17]. Furthermore, other studies have shown a strong association between the timing of motor unit firings and the phase of the vibration cycle by performing intramuscular surface electromyography (s-EMG) [18].

WBV has the following significant advantages: it increases muscular strength [19-21], improves body composition [21, 22] changes muscle performance and balance [23-26], improves human athletic performance such as jumping and running parameters [26,27], and changes the peripheral circulation [28]. According to several studies, WBV exercises significantly increase muscle activity during the exposure with the help of s-EMG recordings [29,30]. According to a previous study, calf muscles experience higher acceleration due to their proximity to the platform, increased reflex activity [31], and/or increased muscle damping [32], further explaining why it has been previously found that the muscles in close proximity to the platform were more affected by WBV than the muscles that are not in close proximity to the platform $[21,33]$. Moreover, vibration exercise generally us- ing the WBV protocols, has been proposed as a potential warm-up modality [34].

When local vibration is applied to the muscle belly or its tendon, several mechanisms have to be considered. First, when vibration is directly applied to the target muscle or tendon, it elicits a reflex muscle contraction [35] that potentially increases muscle function [36]. This particular reflex activity has been referred to as the "tonic vibration reflex", which is the most frequently cited mechanism. Second, local vibration induces cortical activation due to the cortical projection of the muscle afferents [15]. Furthermore, it is also important to note that because vibration is directly applied to the muscle or tendon, a larger vibration amplitude is not required, different from indirect vibration where vibration transmission can be reduced to achieve its target destination by passing through other muscles and joints [37]. Moreover, the cost, portability and localization of vibration are considered to be the potential limitations that may restrict the use of indirect vibration [1]. Considering the limitations of WBV, several local vibrations were considered. The devices used for providing local vibration are powered by batteries, they are portable, and are easy to use; thus, these devices are considered to be beneficial in the current vibration therapy equipment [1]. However, currently, studies assessing the duration, vibration frequency, amplitude of local vibration [1] and its mode of the application are relatively few.

Thus, this study aimed to investigate the immediate changes of concentric peak torque, eccentric peak torque, and muscle activation of the ankle plantar flexor muscle using intermittent local vibration.

\section{Methods}

\section{Participants}

In the beginning, a total of 40 subjects ( 20 males, 20 females) between the age of 20-39 years old volunteered to participate in the experiment from Sahmyook University (Seoul, Korea). The exclusion criteria were as follows: (1) subjects complaining of neurological or vascular symptoms, (2) those with muscular pain or surgery in the calf (3) those with dermatopathy or infectious diseases, (4) those who participated in similar experiments or experienced physical therapy in 3 months on the calf area, and (5) those with reduced cognitive capacity that did not understand the information given about the experiment.

The present study was approved by the Sahmyook University Institutional Review Board (2-7001793-AB- 
$\mathrm{N}-012019106 \mathrm{HR})$. The aim of this study and procedure were informed to the subjects before the experiment, and all participants provided their written consent as follows with the ethical principles of the Declaration of Helsinki.

\section{Experimental procedure and method}

Four subjects were excluded for not meeting the selection criteria, leaving a total of thirty-six subjects for the study. All baseline data of concentric, eccentric peak torque, and muscle activity of the dominant side lateral gastrocnemius muscle were collected for each participant. The peak torque values were collected using an isokinetic device, and the muscle activity values were collected with s-EMG. After the pre-test, an intermittent mode of 1:1 ratio local vibration was activated around the calf for 10 minutes while sitting in the recommended sitting position, and all subjects were instructed to avoid physical activity before and during participation. Post-test values were repeatedly measure the right after the vibration device went off automatically, which was the same exact way as the pre-test values were measured. The trial was implemented for 4 times with submaximal contraction, and completion of 4 repetitions of maximal contraction was recorded for analysis. Between every experiment there was a rest period of 60 seconds. To get accustomed to the experimental procedures, trials of the process were conducted two weeks prior of the study. The participants were assigned to receive the whole similar process and the intervention was performed by one researcher and one assistant who were in charge of measuring s-EMG and were trained to prevent possible accidents during the whole experimental procedure.

\section{Local vibration}

Regarding local vibration, a vibratory device (Myovolt; Christchurch, NewZealand) was positioned on the right calf, which was held in place by a band pro-wrap (Myovolt, Christchurch, NewZealand). During local vibration, participants comfortably sat in a chair with hips and knees flexed at $90^{\circ}$. Based on a previous study [8] and according to the manufacturer's instructions, the 1:1 ratio intermittent pulsing mode device was activated for 10 minutes [8]. The vibration frequency passed through a range of $30-100 \mathrm{~Hz}$ at an amplitude of $0-0.12 \mathrm{~mm}$ in $0.5 \mathrm{~second}$, which created a pulsing effect.

\section{Measurement of muscle activity}

In this study, muscle activation was measured using a wireless s-EMG (2017; Mini Wave Infinity waterproof, Cometa, Italy). Before proceeding with the experiment, all participants' gastrocnemius lateralis muscles were gently abraded and wiped with alcohol swabs. Subsequently, s-EMG was attached to the gastrocnemius lateralis muscle. To reduce the noise of the wireless EMG, an adhesive tape was used to fix the sensor. The muscle activity of the plantar flexor, specifically the gastrocnemius lateralis, was measured using s-EMG while simultaneously measuring both concentric and eccentric contractions. After the preparation has been completely performed, the s-EMG was positioned at a point $1 / 3$ between the fibular head and heel, which was in accordance to the guideline of the SENIAM [38]. All measurements were performed four times. EMG signals were amplified with an amplifier, band-pass filtered, and (analogue to digital) converted. The analog signal from raw data was converted to the American Standard Code for Information Interchange form. Subsequently, the data were sent to a personal computer for editing and signal processing stage using the MyoResearch XP, Noraxon program. The force and EMG data were at a sampling rate of $2 \mathrm{kHz}$ and filtered with a band-pass filter of 20-450 Hz. Four movement contractions were edited separately, and pre-test and post-test data were calculated with the mean values.

\section{Measurement of peak torque}

The isokinetic dynamometer was used to assess the concentric and eccentric peak torques. The positioning of the isokinetic device was based on the positioning of a previous single- group study that examined the acute effects of local Achilles tendon vibration on peak plantar flexor torque, twitch contractile properties, plantar flexor muscle activity, and brain activity [39]. An isokinetic dynamometer (HUMAC, NORM 770; Computer Sports Medicine Inc., Stoughton, MA, USA) was calibrated prior to the start of the study according to the operating manual's instructions. No gravity correction was used for all measurements. The software installed on the computer was similar (HUMAC 2009, v.9.7.1). An isokinetic device was individually adjusted with the participants lying in prone position with hip, knee, and ankle joints in neutral position $\left(180^{\circ}, 180^{\circ}\right.$, and $90^{\circ}$, respectively) [39]. To evoke a more pronounced muscle twitch of the plantar flexor muscle, the muscles around the knee were fully extended (i.e., $180^{\circ}$ ) (i.e., the foot, specifically the heel of the right leg). The leg was firmly attached to the lever arm of the dynamometer with its rotational axis at the level of the malleoli. To limit upper body contributions to torque pro- 
duction, straps were applied at the hip and knee level. This fixed position on the dynamometer was maintained throughout the entire procedure during the familiarization and experimental sessions. The trial was implemented for 4 times, and completion of 4 repetitions was recorded for analysis. This was followed by four concentric and eccentric measurements that were performed at an angular velocity of $60 \% \mathrm{~s}$ with participants in a comfortable position, from $10^{\circ}$ dorsiflexion to $20^{\circ}$ plantarflexion set in range of motion with a resting period of 60 seconds with each repetition. The maximum concentric and eccentric torque values were used for subsequent analysis, which was normalized to the pre-exercise value. During the measurement of each peak torque, verbal encouragement was provided by a similar examiner by verbally instructing the participants to do the following: "contract as forceful and as fast as you can."

\section{Data analysis}

Regarding the statistical analysis, to determine a significant difference after applying local vibration, pre and postmuscle performance and concentric and eccentric values were calculated and assessed using the paired t-test. To obtain a statistical result, all the four-time experiment mean values were used. All statistical analysis was calculated

Table 1. The general characteristics of participants $\quad(\mathrm{N}=36)$

\begin{tabular}{lc}
\hline \multicolumn{1}{c}{ Variable } & Participants \\
\hline Sex $($ male/female $)$ & $16 / 20$ \\
Age $(\mathrm{y})$ & $22.25(4.32)$ \\
Height $(\mathrm{cm})$ & $167.86(7.95)$ \\
Weight $(\mathrm{kg})$ & $62.56(11.97)$ \\
\hline
\end{tabular}

Values are presented as number only or mean (SD).

Table 2. Comparison of muscle activity between pre and posttest $(\mathrm{N}=36)$

\begin{tabular}{lc}
\hline \multicolumn{1}{c}{ Variable } & Participants \\
\hline EMG mean $(\mu \mathrm{V})$ & \\
Before & $314.09(157.97)$ \\
After & $363.21(200.85)$ \\
Difference & $-49.12(141.72)$ \\
$\mathrm{t}(p)$ & $-2.080(0.045)$ \\
EMG peak $(\mu \mathrm{V})$ & \\
Before & $117.54(71.43)$ \\
After & $429.22(224.52)$ \\
Difference & $-311.68(184.50)$ \\
$\mathrm{t}(p)$ & $-10.136(<0.001)$ \\
\hline
\end{tabular}

Values are presented as mean (SD).

EMG: surface electromyography.
IBM SPSS Statistics ver. 22.0 software (IBM Co., Armonk, NY, USA). A significance level was set at a $p$-value of $<0.05$.

\section{Results}

\section{General characteristics of the participants}

Thirty-six participants in this study. The participants' demographic characteristics are shown in Table 1.

\section{Comparison of muscle activity}

The s-EMG results showed significant differences in both mean and peak values $(p<0.05)$. A significant increase in muscle activity was observed in the pre and post-test (Table 2, Figure 1).

\section{Comparison of peak torques}

Descriptive data for the peak torque of concentric and eccentric contractions are presented in Table 3. Our analysis



Figure 1. Comparison of muscle activity between pre and post-test. s-EMG: surface electromyography, white box: pre-test, gray box: post-test. ${ }^{*} p<0.05$.

Table 3. Comparison of peak torque between pre and posttest

$(\mathrm{N}=36)$

\begin{tabular}{lr}
\hline \multicolumn{1}{c}{ Variable } & Participants \\
\hline Concentric peak torque $(\mathrm{Nm})$ & \\
Before & $23.71(17.42)$ \\
After & $31.05(27.77)$ \\
Difference & $-7.33(25.61)$ \\
$\mathrm{t}(p)$ & $-1.718(0.095)$ \\
Eccentric peak torque $(\mathrm{Nm})$ & \\
Before & $42.38(32.02)$ \\
After & $51.97(35.26)$ \\
Difference & $-9.59(22.00)$ \\
$\mathrm{t}(p)$ & $-2.616(0.013)$ \\
\hline
\end{tabular}

Values are presented as mean (SD). 


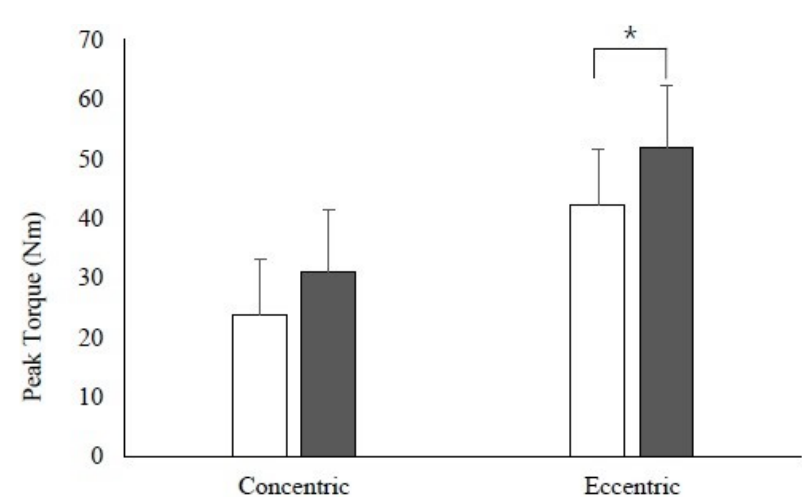

Figure 2. Comparison of peak torque between pre and post-test. White box: pre-test; gray box: post-test. ${ }^{*} p<0.05$.

revealed that a significant difference was observed in eccentric contraction $(p<0.05)$. However, no significant difference was observed in concentric contraction $(p>0.05$ ) (Table 3, Figure 2).

\section{Discussion}

This study aimed to investigate the immediate effects of local vibration on ankle plantar flexor muscle activation and peak torque. The results from this study clearly demonstrate that use of an intermittent mode of local vibration can increase muscle activation and eccentric peak torque. Vibratory stimuli potentially enhance neural and/or muscular (re)conditioning [15]. Local vibration could be an alternative form of vibration training. Local vibration requires the use of a small and portable device that applies vibration directly over the muscle or the tendon [40]. On the contrary, WBV energy from vibration is reduced by the ankle joint, knee joint, and calf musculature, which may influence the magnitude of the vibration stimulus applied to the proximal structures. This reduction in energy from the vibration signal could be lessened if vibrations were applied directly to the muscle of interest via local vibration rather than WBV [36].

First, the present study clearly showed the result of increased muscle activity in both peak value and mean value. This result is consistent with the results of previous several studies. However, each previous study applied various durations. According to a previous study, when vibration was applied to the biceps brachii (frequency range, $0-170 \mathrm{~Hz}$ ) with concentric dynamic biceps curls after 10 minutes in master hockey players, significant increases in power output were observed [5], suggesting that short-term local vibration may have contributed to muscle performance enhancements with contractile properties. Vibration stimulates muscle spindles and activates the $\alpha$-motoneurons [41] and sends a nerve impulse to initiate muscle contractions through a tonic vibration reflex [29].

Second, the findings in this study showed that once the direct pulsing mode vibration was applied on the gastrocnemius lateralis muscle, the eccentric peak torque value showed a significant difference. On the contrary, the concentric peak torque value showed an insignificant difference. Based on a previous study, local vibration was applied for 30 seconds and 300 seconds to the plantar flexor local tendon with a frequency of $80 \mathrm{~Hz}$ and an amplitude of $1 \mathrm{~mm}$. Both durations have not shown any significant difference but showed medium-sized effects of condition with the peak plantar flexor torque [39]. A previous study has focused on the individual's characteristics based on a previous study conducted in athletes [42]. These findings indicate that participants who showed a significant increase in contractile properties are not necessarily those showing the most significant performance improvements following acute exercise [39]. The present study used an isokinetic device set with an angular velocity of $60^{\circ} \mathrm{s}$, from $10^{\circ}$ ankle dorsiflexion to $20^{\circ}$ plantar flexion based on a previous study to measure both the concentric and eccentric peak torque values. This means a single task should be ideally performed in 0.5 seconds, significantly fast to perform. Hence, it was considered to be a difficult task even for healthy nonathletic adults. The result of the present study was inconsistent with the results of the previous studies, specifically differences in placement and types of vibratory devices. Based on a previous study, the vibratory device was placed over the mid-belly of the biceps brachii, and considering its flexible design with the strap and device itself, the muscles applied with vibration can be enclosed. The vibratory panels of the device were placed lateral, medial, superior, and inferior to the center of the muscle [42].

To the best of our knowledge, this is the first study showing a significantly different result regarding the immediate effects of direct pulsing mode vibration on the gastrocnemius lateralis muscle in healthy adults. The result of the present study is consistent with the results of a previous study [5], with increasing peak torque in the elbow flexors. Consistent with the previous studies, the present study used s-EMG and an isokinetic device to determine the muscle activity and peak torque, resulting in unchanged muscle activities after applying vibration to the plantar flexor tendon with different durations such as 30 seconds and 300 seconds 
with a frequency of $80 \mathrm{~Hz}$ [39]. This is possibly attributed to the different methods, vibration types, devices and durations used in the previous studies. Hence, further studies determining the best parameters for local vibration stimuli (e.g., exposure times) in every muscle to create a protocol with acute performance gains are required.

With an increased eccentric peak torque, the s-EMG activity by local vibration on the gastrocnemius lateralis muscle has proven the clear immediate effects of a new portable vibration device. However, this study has the following limitations: The participants were limited to healthy young students, measurement using an isokinetic device may potentially lead to fatigue for the participants, although a 60 -second rest period between every trial was provided, the present study has a limited angular velocity of $60^{\circ} / \mathrm{s}$ to determine the peak torque, and the measurement of pre and post-test data was conducted within the same group and the s-EMG electrodes were not detached during the experiment. Therefore, this study did not use normalization procedures, which has been recommended for inter-individual comparisons [43,44]. Lastly, the present study only showed the results of the immediate effects. Hence, further studies investigating the sustainability of the local vibration effects are required.

In conclusion, vibration device is one of the most commonly used warm-up devices not only for healthy athletes but also for healthy individuals. Therefore, this study was designed to demonstrate the use of local vibration using an intermittent mode to determine its immediate effects on the plantar flexor muscle activation and torque. Devices used in local vibration are portable, powered by batteries, and are easy to use. Based on these results, it can be suggested that local vibration can be possibly considered as one of the effective ways to increase muscle activity and muscle torque, specifically the eccentric peak torque.

\section{Acknowledgements}

This work was supported by the National Research Foundation of Korea (NRF) grant funded by the Korea government (Ministry of Education) (No. 2017R1D1A1B03035062).

\section{Conflict of Interest}

The authors declared no potential conflicts of interest with respect to the authorship and/or publication of this article.

\section{References}

1. Cochrane DJ. Effectiveness of using wearable vibration therapy to alleviate muscle soreness. Eur J Appl Physiol 2017;117:501-9.

2. Stania M, Juras G, Słomka K, Chmielewska D, Król P. The application of whole-body vibration in physiotherapy: a narrative review. Physiol Int 2016;103:133-45.

3. Cardinale M, Wakeling J. Whole body vibration exercise: are vibrations good for you? Br J Sports Med 2005;39:585-9; discussion 9.

4. Ritzmann R, Stark C, Krause A. Vibration therapy in patients with cerebral palsy: a systematic review. Neuropsychiatr Dis Treat 2018;14:1607-25.

5. Cochrane DJ. Does muscular force of the upper body increase following acute, direct vibration? Int J Sports Med 2016;37:54751.

6. Cochrane DJ. Vibration exercise: the potential benefits. Int J Sports Med 2011;32:75-99.

7. Rittweger J. Vibration as an exercise modality: how it may work, and what its potential might be. Eur J Appl Physiol 2010;108: 877-904.

8. Souron R, Zambelli A, Espeit L, Besson T, Cochrane DJ, Lapole T. Active versus local vibration warm-up effects on knee extensors stiffness and neuromuscular performance of healthy young males. J Sci Med Sport 2019;22:206-11.

9. Imtiyaz S, Veqar Z, Shareef MY. To compare the effect of vibration therapy and massage in prevention of delayed onset muscle soreness (DOMS). J Clin Diagn Res 2014;8:133-6.

10. Brunetti O, Filippi GM, Lorenzini M, Liti A, Panichi R, Roscini $\mathrm{M}$, et al. Improvement of posture stability by vibratory stimulation following anterior cruciate ligament reconstruction. Knee Surg Sports Traumatol Arthrosc 2006;14:1180-7.

11. Alghadir AH, Anwer S, Zafar H, Iqbal ZA. Effect of localised vibration on muscle strength in healthy adults: a systematic review. Physiotherapy 2018;104:18-24.

12. Pojskic H, Pagaduan J, Uzicanin E, Babajic F, Muratovic M, Tomljanovic M. Acute effects of loaded whole body vibration training on performance. Asian J Sports Med 2015;6:e24054.

13. Ritzmann R, Kramer A, Bernhardt S, Gollhofer A. Whole body vibration training--improving balance control and muscle endurance. PLoS One 2014;9:e89905.

14. Morel DS, Dionello CDF, Moreira-Marconi E, Brandão-SobrinhoNeto S, Paineiras-Domingos LL, Souza PL, et al. Relevance of whole body vibration exercise in sport: a short review with soccer, diver and combat sport. Afr J Tradit Complement Altern Med 2017;14(4 Suppl):19-27.

15. Souron R, Besson T, Millet GY, Lapole T. Acute and chronic neuromuscular adaptations to local vibration training. Eur J Appl Physiol 2017;117:1939-64.

16. Cardinale M, Bosco C. The use of vibration as an exercise intervention. Exerc Sport Sci Rev 2003;31:3-7.

17. Ritzmann R, Kramer A, Gruber M, Gollhofer A, Taube W. EMG activity during whole body vibration: motion artifacts or stretch reflexes? Eur J Appl Physiol 2010;110:143-51.

18. Pollock RD, Woledge RC, Martin FC, Newham DJ. Effects of whole body vibration on motor unit recruitment and threshold. $\mathrm{J}$ Appl Physiol (1985) 2012;112:388-95. 
19. Delecluse C, Roelants M, Diels R, Koninckx E, Verschueren S. Effects of whole body vibration training on muscle strength and sprint performance in sprint-trained athletes. Int J Sports Med 2005;26:662-8.

20. Delecluse C, Roelants M, Verschueren S. Strength increase after whole-body vibration compared with resistance training. Med Sci Sports Exerc 2003;35:1033-41.

21. Roelants M, Verschueren SM, Delecluse C, Levin O, Stijnen V. Whole-body-vibration-induced increase in leg muscle activity during different squat exercises. J Strength Cond Res 2006;20: 124-9.

22. Vissers D, Verrijken A, Mertens I, Van Gils C, Van de Sompel A, Truijen $\mathrm{S}$, et al. Effect of long-term whole body vibration training on visceral adipose tissue: a preliminary report. Obes Facts 2010; 3:93-100.

23. Runge M, Rehfeld G, Resnicek E. Balance training and exercise in geriatric patients. J Musculoskelet Neuronal Interact 2000;1: 61-5.

24. Torvinen S. Effect of whole body vibration on muscular performance, balance, and bone [Academic dissertation]. Tampere: University of Tampere, Medical school, Department of Surgery; 2003.

25. Torvinen S, Kannu P, Sievänen H, Järvinen TA, Pasanen M, Kontulainen S, et al. Effect of a vibration exposure on muscular performance and body balance. Randomized cross-over study. Clin Physiol Funct Imaging 2002;22:145-52.

26. Torvinen S, Kannus P, Sievänen H, Järvinen TA, Pasanen M, Kontulainen $\mathrm{S}$, et al. Effect of four-month vertical whole body vibration on performance and balance. Med Sci Sports Exerc 2002;34:1523-8

27. Rittweger J, Beller G, Felsenberg D. Acute physiological effects of exhaustive whole-body vibration exercise in man. Clin Physiol 2000;20:134-42.

28. Kerschan-Schindl K, Grampp S, Henk C, Resch H, Preisinger E, Fialka-Moser V, et al. Whole-body vibration exercise leads to alterations in muscle blood volume. Clin Physiol 2001;21:377-82.

29. Cardinale M, Lim J. Electromyography activity of vastus lateralis muscle during whole-body vibrations of different frequencies. J Strength Cond Res 2003;17:621-4.

30. Di Giminiani R, Masedu F, Tihanyi J, Scrimaglio R, Valenti M. The interaction between body position and vibration frequency on acute response to whole body vibration. J Electromyogr Kinesiol 2013;23:245-51.

31. Cochrane DJ, Loram ID, Stannard SR, Rittweger J. Changes in joint angle, muscle-tendon complex length, muscle contractile tissue displacement, and modulation of EMG activity during acute whole-body vibration. Muscle Nerve 2009;40:420-9.

32. Wakeling JM, Kaya M, Temple GK, Johnston IA, Herzog W. Determining patterns of motor recruitment during locomotion. $\mathrm{J}$ Exp Biol 2002;205(Pt 3):359-69.

33. Pollock RD, Woledge RC, Mills KR, Martin FC, Newham DJ. Muscle activity and acceleration during whole body vibration: effect of frequency and amplitude. Clin Biomech (Bristol, Avon) 2010;25:840-6.

34. Cochrane D. The sports performance application of vibration exercise for warm-up, flexibility and sprint speed. Eur J Sport Sci 2013;13:256-71.

35. Eklund G, Hagbarth KE. Normal variability of tonic vibration reflexes in man. Exp Neurol 1966;16:80-92.

36. Pamukoff DN, Pietrosimone B, Lewek MD, Ryan ED, Weinhold PS, Lee DR, et al. Immediate effect of vibratory stimuli on quadriceps function in healthy adults. Muscle Nerve 2016;54:469-78.

37. Luo J, McNamara BP, Moran K. A portable vibrator for muscle performance enhancement by means of direct muscle tendon stimulation. Med Eng Phys 2005;27:513-22.

38. Hermens HJ, Freriks B, Merletti R, Stegeman D, Blok J, Rau G, et al. European recommendations for surface electromyography. Roessingh Research and Development 1999;8:13-54.

39. Zinke F, Gebel A, Granacher U, Prieske O. Acute effects of short-term local tendon vibration on plantar flexor torque, muscle contractile properties, neuromuscular and brain activity in young athletes. J Sports Sci Med 2019;18:327-36.

40. Cochrane DJ. The acute effect of direct vibration on muscular power performance in master athletes. Int J Sports Med 2016;37: 144-8.

41. Lapole T, Pérot C. Effects of repeated Achilles tendon vibration on triceps surae force production. J Electromyogr Kinesiol 2010; 20:648-54.

42. Monazzam MR, Shoja E, Zakerian SA, Foroushani AR, Shoja $\mathrm{M}$, Gharaee $\mathrm{M}$, et al. Combined effect of whole-body vibration and ambient lighting on human discomfort, heart rate, and reaction time. Int Arch Occup Environ Health 2018;91:537-45.

43. Santana-Mora U, López-Ratón M, Mora MJ, Cadarso-Suárez C, López-Cedrún J, Santana-Penín U. Surface raw electromyography has a moderate discriminatory capacity for differentiating between healthy individuals and those with TMD: a diagnostic study. J Electromyogr Kinesiol 2014;24:332-40.

44. Ferrario VF, Sforza C, Colombo A, Ciusa V. An electromyographic investigation of masticatory muscles symmetry in normo-occlusion subjects. J Oral Rehabil 2000;27:33-40. 